\title{
NAGATA RINGS AND DIRECTED UNIONS OF ARTINIAN SUBRINGS
}

\author{
D. KARIM
}

Received 24 October 2002

\begin{abstract}
We investigate when a Nagata ring $R(X)$ can be written as a directed union of Artinian subrings. For a family of zero-dimensional rings $\left\{R_{\alpha}\right\}_{\alpha \in A}$, we show that $\prod_{\alpha \in A} R_{\alpha}\left(X_{\alpha}\right)$ is not a directed sum of Artinian subrings.
\end{abstract}

2000 Mathematics Subject Classification: 13A99, 13A15, 13B02, 13E05.

1. Introduction. All rings considered in this paper are assumed to be commutative with a unit element. If $R$ is a subring of a $\operatorname{ring} S$, we assume that the unity element of $S$ belongs to $R$, and hence is the unit element of $R$. Let $\operatorname{Spec}(R), \mathrm{Z}(R), \operatorname{Inv}(R)$, and $\operatorname{Ann}_{R}(I)$ denote, respectively, the spectrum of $R$ (the set of prime ideals of $R$ ), the set of zero-divisors of $R$, the set of invertible elements of $R$, and the annihilator of a subset $I$ of $R$. By the dimension of $R$, denoted as $\operatorname{dim} R$, we mean the Krull dimension: $\operatorname{dim} R$ is the maximal length of a chain of proper prime ideal $P_{0} \subset P_{1} \subset \cdots \subset P_{n}$ of $R$. If there is no upper bound on the length of such chains, then we write $\operatorname{dim} R=\infty$.

In this paper, we study zero-dimensional rings, in which each proper prime ideal is maximal, and Nagata rings. Our attention will be focused on proving that an infinite direct product of $R_{\alpha}\left(X_{\alpha}\right)$, where $\left\{R_{\alpha}\right\}_{\alpha \in A}$ is a family of zerodimensional rings and $\left\{X_{\alpha}\right\}_{\alpha \in A}$ is a family of indeterminates, is not a directed union of Artinian subrings. Rings of Krull dimension zero have been studied intensively in the literature since the sixties. Directed unions of Artinian subrings have been investigated more recently, see [5, 7].

In [1, Problem 42], Gilmer and Heinzer raised the following question.

(Q) Under which conditions is a von Neumann regular ring a directed union of Artinian subrings?

It would be interesting to consider this question for Nagata rings.

In 1992, Gilmer and Heinzer showed that a product of zero-dimensional rings has dimension zero or infinity, see [6, Theorem 11].

Let $R$ be a commutative ring and $f \in R[X]$. The content of $f$ is the ideal $\sigma(f)$ of $R$ generated by the coefficients of $f$. Then

$$
S=\{f \in R[X] \mid \sigma(f)=R\}=R[X] \backslash \bigcup\{M R[X] \mid M \text { is a maximal ideal of } R\}
$$


is a multiplicatively closed subset of $R[X]$, and the localization $R(X)=$ $S^{-1} R[X]$ is called the Nagata ring in one variable over $R$. The Nagata ring in $n$ variables with coefficients in $R$ is the ring

$$
R(n)=R\left(X_{1}, \ldots, X_{n}\right)=S_{n}^{-1} R\left[X_{1}, \ldots, X_{n}\right]
$$

where

$$
S_{n}=\left\{f \in R\left[X_{1}, \ldots, X_{n}\right] \mid \sigma(f)=R\right\}
$$

If $R$ is zero dimensional, then by [2, Proposition 1.21], $\operatorname{dim} R(X)=\operatorname{dim} R[X]-$ $1=0$ since $\operatorname{dim}(R[X])=1$ (cf. [13, Theorem 2]). Hence $R(X)$ is also zero dimensional.

2. Nagata rings. We first fix notation. Data will consist of a directed system $\left(R_{j}, f_{j k}\right)$ of rings indexed by a directed set $(I, \leq)$ and its directed union $R=$ $\bigcup_{j \in I} R_{j}$, together with the canonical maps $f_{j}: R_{j} \rightarrow R$. The ring $R$ is a directed union of $R_{j}$ 's corresponding to the $f_{j k}$ 's being inclusion maps. Thus directed unions can be treated by assuming all $f_{j k}$ to be monomorphisms. Notice that if $R_{j}$ is a ring for each $j \in I$, then $R$ is also a ring. However, $R$ is not necessarily Noetherian even if each $R_{j}$ is Noetherian. If $R=\bigcup_{j \in I} R_{j}$ is a directed union of Artinian subrings, then we regard each $R_{i}$ as a subring of $R$; in particular, $R_{i}$ and $R$ have the same unit element.

The proof of Lemma 2.1 is straightforward and is left to the reader.

LEMMA 2.1. Let $\left\{R_{i}\right\}_{i=1}^{k}$ be a finite family of rings and $X$ a variable. Then $\left(\prod_{i=1}^{k} R_{i}\right)[X] \simeq \prod_{i=1}^{k} R_{i}[X]$.

Let $K$ and $L$ be two fields. From Lemma 2.1, we know that $(K \times L)[X]$ is isomorphic to $K[X] \times L[X]$, and we can view a polynomial in $(K \times L)[X]$ as a pair of polynomials. Take $g / h \in(K \times L)(X)$. Then $g, h \in(K \times L)[X]$ such that $\sigma(h)=K \times L$. We have $g=\left(g_{1}, g_{2}\right)$, where $g_{1} \in K[X]$ and $g_{2} \in L[X]$; also $h=\left(h_{1}, h_{2}\right)$ such that $h_{1} \in K[X] \backslash(0)$ and $h_{2} \in L[X] \backslash(0)$. Hence $g / h=$ $\left(g_{1} / h_{1}, g_{2} / h_{2}\right) \in K(X) \times L(X)$. Therefore, we have $K(X) \times L(X) \simeq(K \times L)(X)$, and hence we have the following result.

Proposition 2.2. Let $\prod_{i=1}^{n} K_{i}$ be a finite product of fields $K_{i}$. Then $\left(\prod_{i=1}^{n} K_{i}\right)(X) \simeq \prod_{i=1}^{n} K_{i}(X)$ is a finite product of fields.

Proposition 2.3. If $\left\{R_{\alpha}\right\}_{\alpha \in A}$ is an infinite family of rings and $X$ a variable over $\prod_{\alpha \in A} R_{\alpha}$, then $\varphi:\left(\prod_{\alpha \in A} R_{\alpha}\right)(X) \rightarrow \prod_{\alpha \in A} R_{\alpha}(X)$ is an injective homomorphism.

Before proving Proposition 2.3, we need the following lemma. 
LEMMA 2.4. If $f=\left(f_{\alpha}\right)_{\alpha \in A} \in \prod_{\alpha \in A} R_{\alpha}[X]$, then $\sigma(f)=\prod_{\alpha \in A} \sigma\left(f_{\alpha}\right)$.

Proof. Let $f=h_{n} X^{n}+\cdots+h_{1} X+h_{0}$, with $h_{i} \in \prod_{\alpha \in A} R_{\alpha}$ for each $i=$ $0,1, \ldots, n$. Then $\sigma(f)=\left(h_{n}, \ldots, h_{1}, h_{0}\right) \prod_{\alpha \in A} R_{\alpha}$, which implies that $\sigma\left(f_{\alpha}\right)=$ $\left(h_{n}(\alpha), \ldots, h_{1}(\alpha), h_{0}(\alpha)\right) R_{\alpha}$, where $h_{i}(\alpha)$ is the $\alpha$ th component of $h_{i}$. We can conclude that $\sigma(f) \subseteq \prod_{\alpha \in A} \sigma\left(f_{\alpha}\right)$. The proof of the converse is similar.

Proof of Proposition 2.3. The map $\varphi:\left(\prod_{\alpha \in A} R_{\alpha}\right)[X] \rightarrow \prod_{\alpha \in A} R_{\alpha}[X]$ defined by $\varphi(F)=(F(\alpha))_{\alpha \in A}$, where $F(\alpha)$ is the polynomial projection of $F$ over $R_{\alpha}[X]$, is an injective homomorphism. Now, we consider

$$
\psi: s\left(\prod_{\alpha \in A} R_{\alpha}\right)(X) \longrightarrow \prod_{\alpha \in A} R_{\alpha}(X)
$$

defined by $\psi(F / H)=(F(\alpha) / H(\alpha))_{\alpha \in A}$, where $F, H \in\left(\prod_{\alpha \in A} R_{\alpha}\right)[X]$ such that $\sigma(H)=\prod_{\alpha \in A} R_{\alpha}$. It is clear that $\psi$ is a homomorphism. Now, let $F / H \in$ $\left(\prod_{\alpha \in A} R_{\alpha}\right)(X)$ such that $\psi(F / H)=0$. We have $\sigma(H)=\prod_{\alpha \in A} R_{\alpha}$, and, by Lemma 2.4, $\sigma(H(\alpha))=R_{\alpha}$. Then $\psi(F / H)=(F(\alpha) / H(\alpha))_{\alpha \in A}=0$ implies that $F(\alpha) / H(\alpha)=0$ in $R_{\alpha}(X)$ for each $\alpha \in A$. For each $\alpha \in A$, there exists $h_{\alpha} \in$ $R_{\alpha}[X]$ such that $\sigma\left(h_{\alpha}\right)=R_{\alpha}$ and $F(\alpha) h_{\alpha}=0$. We set $G=\left(h_{\alpha}\right)_{\alpha \in A}$. Then $F G=0$, $G \in\left(\prod_{\alpha \in A} R_{\alpha}\right)[X]$, and, by Lemma 2.4, $\sigma(G)=\prod_{\alpha \in A} \sigma\left(h_{\alpha}\right)=\prod_{\alpha \in A} R_{\alpha}$. It follows that $F / H=0$.

The converse of Proposition 2.3 is not true in general, as shown in the next example.

EXAMPLE 2.5. For each $i \in \mathbb{Z}^{+}$, let $R_{i}=\mathbb{F}_{p^{i}}$ be the Galois field with $p^{i}$ elements, where $p$ is a positive prime integer. From [10, Theorem 5.5, page 247], $\mathbb{F}_{p^{i}}=\operatorname{GF}(p)\left(\xi_{i}\right)$, where $\xi_{i}$ is a $p^{i}$ th primitive root of unity, for each $i \in \mathbb{Z}^{+}$, and $\operatorname{GF}(p)$ is the Galois field with $p$ elements. Let $\xi=\left\{\xi_{i}\right\}_{i=1}^{\infty}$ be an element of $\prod_{i=1}^{\infty} R_{i}$ such that $\left[\mathrm{GF}(p)\left(\xi_{i}\right): \mathrm{GF}(p)\right]=i$, for each $i \in \mathbb{Z}^{+}$, and $f_{i}=\operatorname{Irr}\left(\xi_{i}, \mathrm{GF}(p)\right)$ the minimal polynomial of $\xi_{i}$ over $\mathrm{GF}(p)$. Clearly, $f_{i}$ is of degree $i$. It follows that there exists no monic polynomial $F \in \prod_{i=1}^{\infty} R_{i}[X]$ such that $F=\left\{f_{i}\right\}_{i=1}^{\infty}$, otherwise $\xi$ is an integral element over $\prod_{i=1}^{\infty} \mathrm{GF}(p)$ since $F(\xi)=0$.

Our next result will be useful later.

LEMMA 2.6. Let $R$ be a zero-dimensional ring with finite spectrum. Then $R$ can be expressed as a finite product of zero-dimensional quasilocal subrings.

Proof. Let $\operatorname{Spec}(R)=\left\{M_{i}\right\}_{i=1}^{n}$ be the spectrum of $R$. Let $S_{M_{i}}(0)=\operatorname{Ker} \varphi_{i}$, for each $i=1, \ldots, n$, where $\varphi_{i}: R \rightarrow R_{M_{i}}$ and $\varphi_{i}(r)=r / 1$ is the canonical homomorphism. Since $\operatorname{Rad}\left(S_{M_{i}}(0)\right)=M_{i} \in \operatorname{Max}(R)$, then $S_{M_{i}}(0)$ is a primary ideal. Note that $\cap_{i=1}^{n} S_{M_{i}}(0)=(0)$ and $S_{M_{i}}(0)+S_{M_{j}}(0)=R$ for each $i \neq j$ in $\{1, \ldots, n\}$. Therefore, $R \simeq R / \cap_{i=1}^{n} S_{M_{i}}(0)$. By the Chinese remainder theorem, 
$R \simeq \prod_{i=1}^{n} R / S_{M_{i}}(0)$, where $R / S_{M_{i}}(0)$ is quasilocal and zero-dimensional, for $i=1, \ldots, n$.

We note that if $R$ is a von Neumann regular ring (i.e., $R$ is reduced and zero dimensional), then $R$ is an Artinian if and only if $R$ is a finite product of fields if and only if $R$ is Noetherian. Indeed, if $R$ is von Neumann regular and Artinian, then, by [3, Corollary 8.2], $\operatorname{Spec}(R)$ is finite, and hence, $R=R_{1} \oplus \cdots \oplus R_{n}$, where each $R_{i}$ is a quasilocal and zero-dimensional ring, for $i=1, \ldots, n$. Since $R$ is a von Neumann regular ring, each $R_{i}$ is a von Neumann regular ring, by [4, Result 3.2]. As $R_{i}$ is a quasilocal ring, by [4, Theorem 3.1], $R_{i}$ is a field for $i=1, \ldots, n$, and it follows that $R$ is a finite product of fields.

Proposition 2.7. Let $R$ be a ring and $X$ a variable over $R$. Then

(1) if $R$ is a directed union of Artinian subrings, then so is $R(X)$;

(2) if $R$ is a reduced ring and $R(X)$ is a directed union of Artinian subrings, then $R$ has the same property;

(3) $R$ is a directed union of zero-dimensional subrings with finite spectra if and only if $R(X)$ has the same property.

Proof. (1) If $R=\bigcup_{i \in I} R_{i}$ is a directed union of Artinian subrings, then $R(X)=\bigcup_{i \in I} R_{i}(X)$. Since each $R_{i}$ is Noetherian, by [12, (6.17)], $R_{i}(X)$ is also Noetherian and each $R_{i}(X)$ is zero dimensional since each $R_{i}$ is Noetherian, (cf. [2, Proposition 1.21]). By [3, Theorem 8.5], $R_{i}(X)$ is an Artinian ring for each $i \in I$. The family $\left\{R_{i}(X)\right\}_{i \in I}$ is directed because the family $\left\{R_{i}\right\}_{i \in I}$ is directed. Then $R(X)$ is a directed union of Artinian subrings.

(2) If $R(X)$ is a directed union of Artinian subrings, then, by [8, Theorem 2.4(a)], each $R_{j}=S_{j} \cap R$ is zero dimensional. Since $R_{j} \subseteq S_{j}$ and $\operatorname{Spec}\left(S_{j}\right)$ is finite (cf. [3, Theorem 8.3]), this yields that each $\operatorname{Spec}\left(R_{j}\right)$ is finite. As $R$ is reduced, and by [4, Theorem 3.1], each $R_{j}$ is a von Neumann regular ring with finite spectrum. It follows that $R_{j}$ is Artinian, and hence, $R=\bigcup_{j \in I} R_{j}$ is a directed union of Artinian subrings.

(3) The proof of this result follows from the fact that $\operatorname{Spec}(R)=\{\mathfrak{m}(X) \mid \mathfrak{m} \in$ $\operatorname{Spec}(R)\}$, and hence, $|\operatorname{Spec}(R)|=|\operatorname{Spec}(R(X))|$.

REMARK 2.8. (1) Let $R$ be a hereditary zero-dimensional ring, that is, a ring for which all subrings are zero dimensional. Then $R$ is a directed union of Artinian subrings, and therefore, $R(X)$ is a directed union of Artinian subrings that is not hereditarily zero dimensional, since $R[X] \subset R(X)$ and $\operatorname{dim}(R[X])$ $=1$ (cf. [13, Theorem 2]).

(2) Let $R$ be a von Neumann regular ring and $X_{1}, \ldots, X_{n}$ variables over $R$. We denote $R\left(X_{1}, \ldots, X_{n}\right)=R(n)$ for each $n \in \mathbb{Z}^{+}$. Then, by Proposition 2.7 and [9, Lemma 15.3], $R$ is a directed union of Artinian subrings if and only if $R(n)$ is a directed union of Artinian subrings, for each $n \in \mathbb{Z}^{+}$.

(3) If, in Proposition 2.7(3), we take $X=\left\{Y_{i}\right\}_{i \in I}$ an infinite family of indeterminates over $R$, then $R$ is a directed union of zero-dimensional subrings with finite spectra if and only if so is $R(X)$. 
Let $\eta(x)$ be the index of nilpotency of $x \in R$. We define

$$
\eta(R)=\sup \{\eta(x) \mid x \in N(R)\}
$$

where $N(R)$ is the set of nilpotent elements of $R$. From [7, Theorem 3.4], we know that $\operatorname{dim}\left(\prod_{\alpha \in A} T_{\alpha}\right)=0$ if and only if $\left\{\alpha \in A \mid \eta\left(T_{\alpha}\right)>k\right\}$ is finite for some $k \in \mathbb{Z}^{+}$, where $\left\{T_{\alpha}\right\}_{\alpha \in A}$ is a family of zero-dimensional rings.

Let $R$ be a ring such that $\eta(R)<k$ for some $k \in \mathbb{Z}^{+}$and let $X$ be a variable over $R$. Then $\eta(R[X])$ need not be bounded. Also, we note that if $\operatorname{dim}(R)=0$, then $\operatorname{dim}(R(X))=0$.

Let $\left\{R_{\alpha}\right\}_{\alpha \in A}$ be a family of zero-dimensional rings and $X$ a variable, and suppose that $\operatorname{dim}\left(\prod_{\alpha \in A} R_{\alpha}\right)=0$. We added that, if each $R_{\alpha}$ is a directed union of Artinian subrings, then $R_{\alpha}(X)$ is also a directed union of Artinian subrings for each $\alpha \in A$, see Proposition 2.7. Assume that there exists $k \in \mathbb{Z}^{+}$such that $\left\{\alpha \in A \mid \eta\left(R_{\alpha}\right)>k\right.$ or there exists $\left.M \in \operatorname{Spec}\left(R_{\alpha}\right):\left|R_{\alpha} / M\right|>k\right\}$ is finite. Then, by [7, Theorem 6.7], $\prod_{\alpha \in A} R_{\alpha}$ is a directed union of Artinian subrings. However, for each $k \in \mathbb{Z}^{+},\left\{\alpha \in A \mid \eta\left(R_{\alpha}(X)\right)>k\right\}$ is an infinite set. This means that $\prod_{\alpha \in A} R_{\alpha}(X)$ is not zero dimensional. Now, we suppose that each $R_{\alpha}$ is a von Neumann regular ring, and we show that $R_{\alpha}(X)$ is also a von Neumann regular ring, and hence, $\prod_{\alpha \in A} R_{\alpha}\left(X_{\alpha}\right)$ is a von Neumann regular ring, where each $X_{\alpha}$ is a variable over $R_{\alpha}$.

THEOREM 2.9. Let $\left\{R_{\alpha}\right\}_{\alpha \in A}$ be a family of von Neumann regular rings and $X_{\alpha}$ an indeterminate over $R_{\alpha}$, for each $\alpha \in A$. Then $\prod_{\alpha \in A} R_{\alpha}\left(X_{\alpha}\right)$ is not a directed union of Artinian subrings.

The proof of Theorem 2.9 requires the following two lemmas.

LEMMA 2.10. Let $R$ be a ring and $U$ a multiplicatively closed subset of $R$. If $R$ is reduced, then $U^{-1} R$ is also reduced.

Proof. Let $r / s \in N\left(U^{-1} R\right)$, where $N\left(U^{-1} R\right)$ is the nilradical of $U^{-1} R$. Then there exists $n_{0} \in \mathbb{N}^{*}$ such that $(r / s)^{n_{0}}=0$; this means that there exists $u \in U$ such that $(r u)^{n_{0}}=0$. Since $R$ is reduced, we have $r u=0$, and hence, $r / s=0$. In other words, $N\left(U^{-1} R\right)=(0)$. Thus, $U^{-1} R$ is reduced.

LEMMA 2.11. Let $R$ be a ring and $X$ a variable over $R$. Then $R$ is reduced if and only if $R(X)$ is reduced.

Proof. Assume that $R$ is reduced and take $f=a_{n} X^{n}+\cdots+a_{1} X+a_{0} \in$ $N(R[X])$. Then there exists $l \in \mathbb{Z}^{+}$such that $f^{l}=0$. Therefore, $a_{n}^{l}=a_{n-1}^{l}=$ $\cdots=a_{0}^{l}=0$. Since $R$ is reduced, we have $a_{n}=a_{n-1}=\cdots=a_{0}=0$, and hence, $f=0$. It follows that $N(R[X])=(0)$. By Lemma 2.10, $R(X)$ is a also reduced because $R(X)=S^{-1} R[X]$ is a localization of $R[X]$.

The converse implication follows from the fact that every subring of a reduced ring is reduced. 
Note that the two equivalent conditions of Lemma 2.11 are also equivalent to $R(n)$ being reduced since $R(n)=R(n-1)(X)$, for each $n \in \mathbb{Z}^{+}$, see [9, Lemma 15.3].

PROOF OF THEOREM 2.9. By [7, Theorem 6.7], $\prod_{\alpha \in A} R_{\alpha}\left(X_{\alpha}\right)$ is a directed union of Artinian subrings if and only if there exists $k \in \mathbb{Z}^{+}$such that $\{\alpha \in A \mid$ there exists $M \in \operatorname{Spec}\left(R_{\alpha}\left(X_{\alpha}\right)\right)$ with $\left.\left|R_{\alpha} / M\right|>k\right\}$ is finite. It was shown in [12, (6.17)], that $\operatorname{Spec}\left(R_{\alpha}\left(X_{\alpha}\right)\right)=\left\{M\left(X_{\alpha}\right) \mid M \in \operatorname{Spec}\left(R_{\alpha}\right)\right\}$ for each $\alpha \in A$. Moreover, $R_{\alpha}\left(X_{\alpha}\right) / M_{\alpha}\left(X_{\alpha}\right) \simeq\left(R_{\alpha} / M_{\alpha}\right)(X)$ and $\left(R_{\alpha} / M_{\alpha}\right)\left(X_{\alpha}\right)$ is an at least countably denumerable field. Therefore, for each $\alpha \in A$, for each $M_{\alpha} \in \operatorname{Spec}\left(R_{\alpha}\right)$, for each $k \in \mathbb{Z}^{+}$we have $\left|\left(R_{\alpha} / M_{\alpha}\right)\left(X_{\alpha}\right)\right|>k$. Then, for each $k \in \mathbb{Z}^{+}$, the set $\left\{\alpha \in A \mid\right.$ there exists $M_{\alpha} \in \operatorname{Spec}\left(R_{\alpha}\right)$ with $\left.\left|\left(R_{\alpha} / M_{\alpha}\right)\left(X_{\alpha}\right)\right|>k\right\}$ is infinite. Thus, $\prod_{\alpha \in A} R_{\alpha}\left(X_{\alpha}\right)$ is not a directed union of Artinian subrings.

COROLLARY 2.12. Let $\left\{R_{\alpha}\right\}_{\alpha \in A}$ be a family of von Neumann regular rings and $X$ a variable. Then $\prod_{\alpha \in A} R_{\alpha}(X)$ is not a directed union of Artinian subrings.

Let $R$ be a ring and $\left\{R_{\alpha}\right\}_{\alpha \in A}$ an infinite family of nonzero rings such that $R$ is, up to isomorphism, a subring of each $R_{\alpha}$. We use $R^{*}$ to denote the image of $R$ under the diagonal imbedding, that is, $R^{*}=\varphi(R)$, where $\varphi: R \hookrightarrow \prod_{\alpha \in A} R_{\alpha}$ is the monomorphism defined by $\varphi(x)=\left\{x_{\alpha}\right\}_{\alpha \in A}$ such that $x_{\alpha}=x$ for each $\alpha \in A$.

Proposition 2.13. Let $F$ be an absolutely algebraic field and $R=\prod^{\omega_{0}} F$ a countable direct product of copies of $F$. Define

$$
\mathscr{S}=\left\{\left\{x_{i}\right\}_{i=1}^{\infty} \in R \mid\left\{x_{i}\right\}_{i=1}^{\infty} \text { has only finitely many distinct coordinates }\right\} .
$$

Then 9 is the maximal subring of $R$ which can be expressed as a directed union of Artinian subrings.

Proof. First, we claim that $\mathscr{S}$ is a directed union of Artinian subrings. For each $j \in \mathbb{Z}^{+}$, we define $S_{j}$ as the subring of $\mathscr{S}$ consisting of all sequences $\left\{x_{i}\right\}_{i=0}^{\infty} \in \mathscr{Y}$ such that $x_{j}=x_{j+1}=\cdots$. If we denote by $\pi$ the prime subring of $R$, then each $S_{j}$ contains $\pi, S_{0}$ is the diagonal imbedding of $F$ in $R$, and $S_{j} \simeq F^{j+1}$ is an Artinian von Neumann regular ring. Clearly, $S_{j} \subseteq S_{j+1}$ for each $j \in \mathbb{Z}^{+}$. Therefore, $\mathscr{Y}=\bigcup_{j=1}^{\infty} S_{j}$ and $\mathscr{Y}$ is a directed union of Artinian subrings. Now, let $T$ be a subring of $\prod^{\omega_{0}} F$ with $T=\bigcup_{j \in J} T_{j}$ a directed union of Artinian subrings and $t=\left\{t_{i}\right\}_{i=1}^{\infty} \in T$. There exists $j_{0} \in J$ such that $t \in T_{j_{0}}$ and $T_{j_{0}}$ is a finite product of fields; hence $t \in \mathscr{S}$.

EXAMPLE 2.14. Let $p$ be a prime integer and $X$ a variable over $\operatorname{GF}(p)$, where $\mathrm{GF}(p)$ is the Galois field with $p$ elements. Let $R=(\mathrm{GF}(p)(X))^{\omega_{0}}$ be a countable direct product of copies of $\mathrm{GF}(p)(X)$. We note that $R$ is a von Neumann regular ring as a direct product of fields. By Theorem 2.9, the ring $R$ is not a directed union of Artinian subrings. Let $\mathscr{S}=\left\{\left\{x_{i}\right\}_{i=1}^{\infty} \in R:\left\{x_{i}\right\}_{i=1}^{\infty}\right.$ has only finitely many distinct components $\}$ and $V=(\mathrm{GF}(p)(X))^{*}+I$, where $I=\bigoplus_{i=1}^{\infty} \mathrm{GF}(p)$ 
is the direct sum ideal of $R$ and $(\mathrm{GF}(p)(X))^{*}$ is the diagonal imbedding of $\mathrm{GF}(p)(X)$ in $R$. The ring $\mathscr{Y}$ is the biggest subring of $R$ which is a directed union of Artinian subrings. We remark that, if $V \subset \mathscr{S}$, then, by [11, Corollary 4], $V$ is also a directed union of Artinian subrings. Since $\mathrm{GF}(p)$ is a finite field, we have $\prod^{\omega_{0}} \mathrm{GF}(p) \subset \mathscr{S}$ and, by [11, Corollary 4], is a directed union of Artinian subrings.

ACKNOwLEDGment. The author would like to thank the referee for a number of helpful comments and suggestions.

\section{REFERENCES}

[1] D. D. Anderson, Some problems in commutative ring theory, Zero-Dimensional Commutative Rings (Knoxville, Tennesse, 1994) (D. F. Anderson and D. E. Dobbs, eds.), Lecture Notes in Pure and Appl. Math., vol. 171, Marcel Dekker, New York, 1995, pp. 363-372.

[2] D. F. Anderson, A. Bouvier, D. E. Dobbs, M. Fontana, and S. Kabbaj, On Jaffard domains, Exposition. Math. 6 (1988), no. 2, 145-175.

[3] M. F. Atiyah and I. G. Macdonald, Introduction to Commutative Algebra, AddisonWesley, Massachusetts, 1969.

[4] R. Gilmer, Background and preliminaries on zero-dimensional rings, ZeroDimensional Commutative Rings (Knoxville, Tennesse, 1994) (D. F. Anderson and D. E. Dobbs, eds.), Lecture Notes in Pure and Appl. Math., vol. 171, Marcel Dekker, New York, 1995, pp. 1-13.

[5] _ Zero-dimensional subrings of commutative rings, Abelian Groups and Modules (Padova, 1994) (A. Facchini and C. Menini, eds.), Math. Appl., vol. 343, Kluwer Academic, Dordrecht, 1995, pp. 209-219.

[6] _ Zero-dimensionality and products of commutative rings, Zero-Dimensional Commutative Rings (Knoxville, Tennesse, 1994) (D. F. Anderson and D. E. Dobbs, eds.), Lecture Notes in Pure and Appl. Math., vol. 171, Marcel Dekker, New York, 1995, pp. 15-25.

[7] R. Gilmer and W. Heinzer, Products of commutative rings and zero-dimensionality, Trans. Amer. Math. Soc. 331 (1992), no. 2, 663-680.

[8] _ Zero-dimensionality in commutative rings, Proc. Amer. Math. Soc. 115 (1992), no. 4, 881-893.

[9] J. A. Huckaba, Commutative Rings with Zero Divisors, Monographs and Textbooks in Pure and Applied Mathematics, vol. 117, Marcel Dekker, New York, 1988.

[10] S. Lang, Algebra, Addison-Wesley, Massachusetts, 1993.

[11] A. R. Magid, Direct limits of finite products of fields, Zero-Dimensional Commutative Rings (Knoxville, Tennesse, 1994) (D. F. Anderson and D. E. Dobbs, eds.), Lecture Notes in Pure and Appl. Math., vol. 171, Marcel Dekker, New York, 1995, pp. 299-305.

[12] M. Nagata, Local Rings, Interscience Tracts in Pure and Applied Mathematics, no. 13, Interscience, New York, 1962.

[13] A. Seidenberg, A note on the dimension theory of rings, Pacific J. Math. 3 (1953), 505-512.

D. Karim: Department of Mathematics, Faculty of Sciences Semlalia, University of Cadi Ayyad, P.O. Box 2390 Marrakech, Morocco

E-mail address: ikarim@ucam.ac.ma 


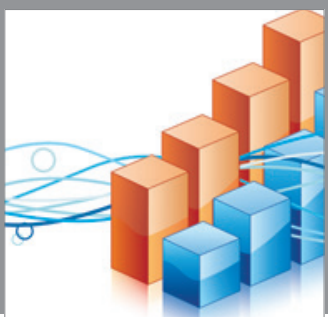

Advances in

Operations Research

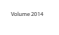

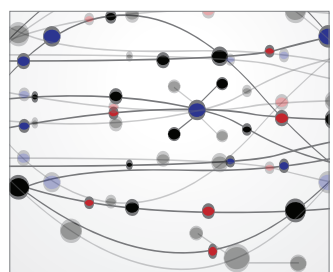

\section{The Scientific} World Journal
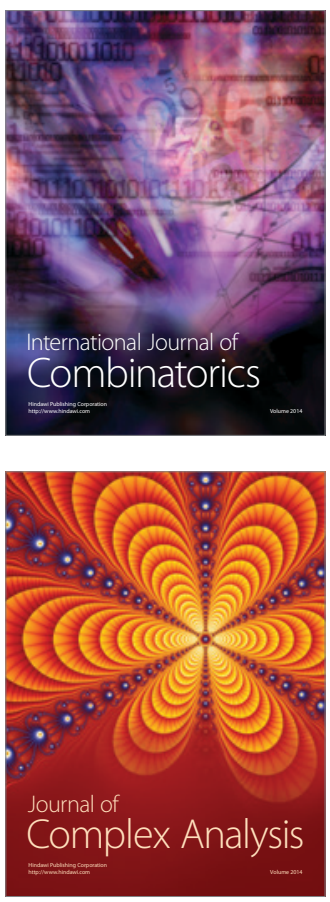

International Journal of

Mathematics and

Mathematical

Sciences
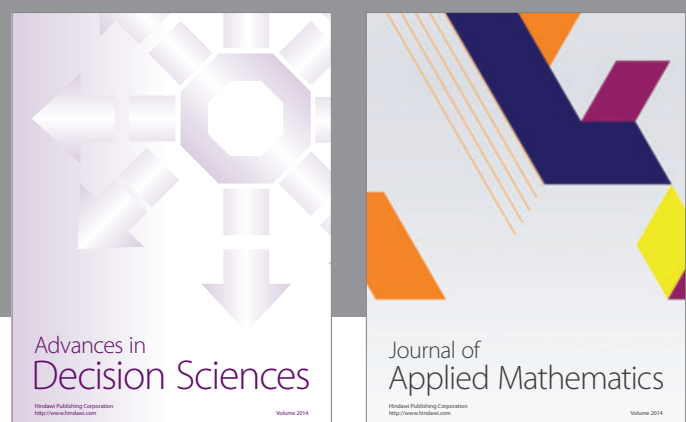

Journal of

Applied Mathematics
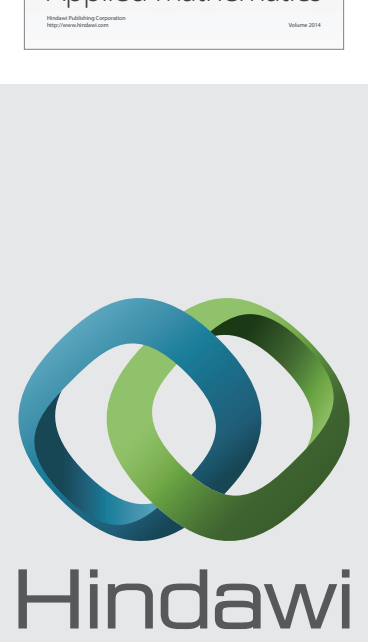

Submit your manuscripts at http://www.hindawi.com
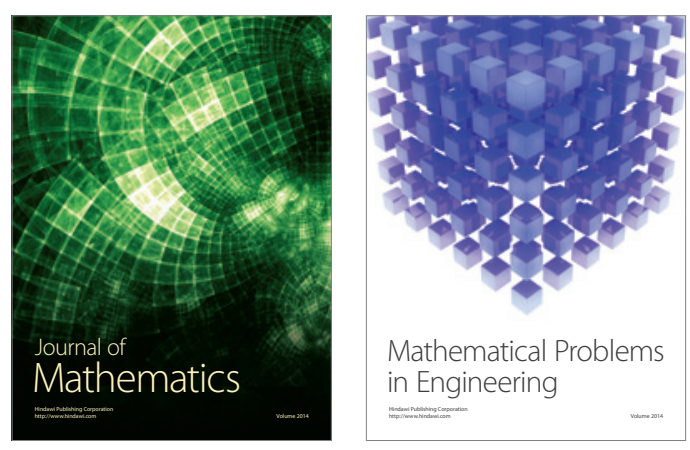

Mathematical Problems in Engineering
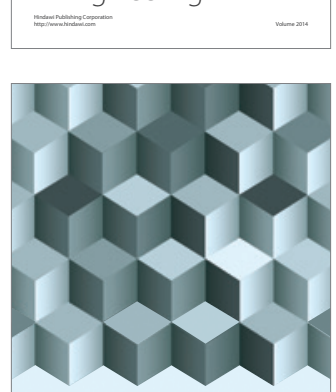

Journal of

Function Spaces
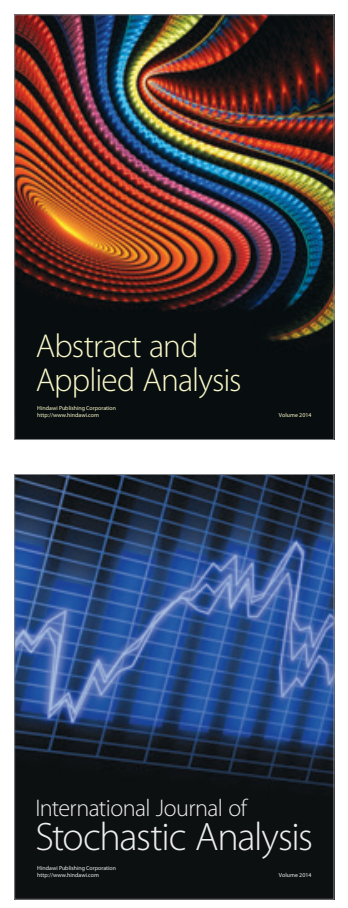

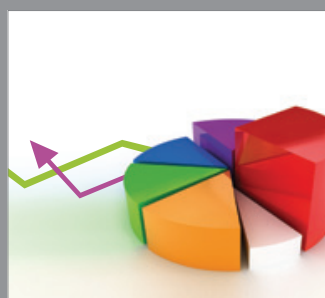

ournal of

Probability and Statistics

Promensencen
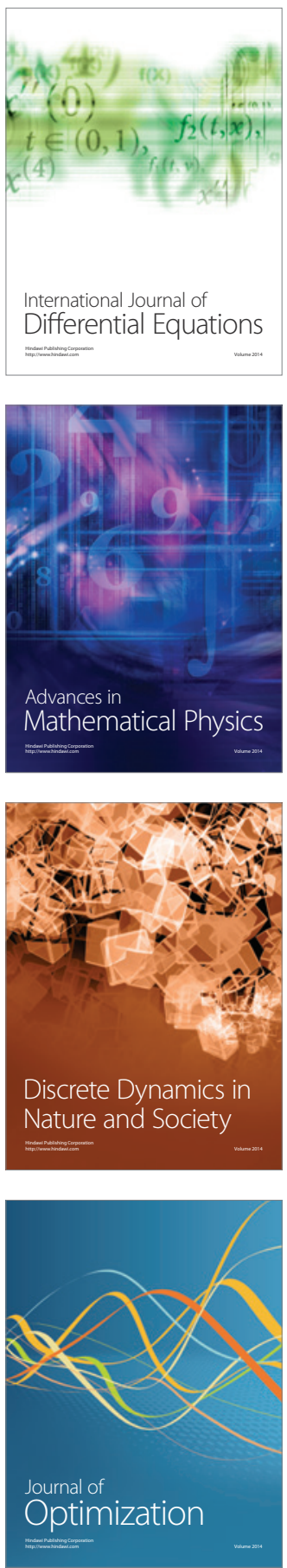